\title{
Estatísticas de avaliação da precisão experimental em ensaios de cultivares de milho
}

\author{
Alberto Cargnelutti Filho(1) e Lindolfo Storck $^{(2)}$
}

\begin{abstract}
(1)Universidade Estadual Paulista, Dep. de Ciências Exatas, Fac. de Ciências Agrárias e Veterinárias, Via de Acesso Prof. Paulo Donato Castellane, s/no, CEP 14884-900 Jaboticabal, SP. E-mail: cargnelutti@fcav.unesp.br (2)Universidade Federal de Santa Maria, Dep. de Fitotecnia, Av. Roraima, s/no, Bairro Camobi, CEP 97105-900 Santa Maria, RS. E-mail: lindolfo@smail.ufsm.br
\end{abstract}

\begin{abstract}
Resumo - Foram usados dados de produtividade de grãos, de 101 ensaios de competição de cultivares de milho (Zea mays L.), realizados no Estado do Rio Grande do Sul, nos anos agrícolas 2002/2003, 2003/2004 e 2004/2005, com o objetivo de avaliar estatísticas para a classificação da precisão experimental. O coeficiente de variação e a diferença mínima significativa, entre as cultivares pelo teste de Tukey expresso em porcentagem da média, estão associadas à média e à variância residual e são estatísticas adequadas para a classificação de experimentos com médias semelhantes. Os maiores valores das estatísticas herdabilidade, coeficiente de determinação, valor do teste F para cultivar, e o índice de diferenciação de Fasoulas estão associados a maiores variabilidades genéticas e menores variâncias residuais, e independem da média do ensaio; estas estatísticas são adequadas para a classificação da precisão de experimentos.
\end{abstract}

Termos para indexação: Zea mays, indicação, coeficiente de variação.

\section{Evaluation statistics of the experimental precision in corn cultivar trials}

\begin{abstract}
Grain yield data were used from 101 cultivar competition trials of corn (Zea mays L.), carried out in Rio Grande do Sul State, Brazil, in the agricultural years 2002/2003, 2003/2004 and 2004/2005, with the aim at evaluating statistics for classification of the experimental precision. The experimental coefficient of variation and significant minimum difference among cultivars by Tukey's test, expressed in percentage of the mean, are associated to the mean and the residual variance, being statistics appropriated for the classification of experiments with similar means. The highest values of the statistics herdability, coefficient of determination, test $\mathrm{F}$ value for cultivars, and Fasoulas index of differentiation are associated to greatest genetic variabilities and minor residual variances, and are independent from the mean trials; these statistics are appropriated for classification of the experimental precision.
\end{abstract}

Index terms: Zea mays, indication, coefficient of variation.

\section{Introdução}

A avaliação da precisão experimental (qualidade) dos resultados de um ensaio de competição de cultivares, e de outros ensaios, é importante para a validação das conclusões obtidas. Em processos de controle de qualidade, o objetivo é o de descartar as entradas de baixa precisão.

O coeficiente de variação $(\mathrm{CV})$ constituiu-se numa estimativa do erro experimental, em relação à média geral do ensaio, e é uma estatística muito utilizada como medida de avaliação da qualidade experimental. Considera-se que quanto menor for a estimativa do CV, maior será a precisão do experimento e vice-versa, e, quanto maior a precisão (maior qualidade) experimental, menores diferenças entre estimativas de médias serão significativas.

Ao estudar os CV de ensaios agrícolas, PimentelGomes (1990) classificou-os da seguinte forma: baixos, quando inferiores a 10\%; médios, entre 10 e $20 \%$; altos, quando estão entre 20 e $30 \%$; e muito altos, quando são superiores a $30 \%$. Essa classificação tem sido extensivamente utilizada, porém não considera as particularidades da cultura avaliada e, principalmente, $\mathrm{o}$ caráter estudado (Garcia, 1989).

Trabalhos relacionados ao estabelecimento de valores críticos de $\mathrm{CV}$, para controle de qualidade de ensaios, e novos critérios ou métodos de classificar a precisão de 
experimentos, com base nos $\mathrm{CV}$ de conjunto de ensaios semelhantes, têm sido desenvolvidos em diversas culturas, tipos de experimentos e caracteres (Estefanel et al., 1987; Garcia, 1989; Pimentel-Gomes, 1991; Scapim et al., 1995; Amaral et al., 1997; Lúcio et al., 1997, 1999; Judice et al., 1999, 2002; Lúcio \& Storck, 1999; Clemente \& Muniz, 2002; Costa et al., 2002; Storck et al., 2002; Carvalho et al., 2003; Lima et al., 2004). De maneira geral, nestes estudos, os valores críticos de precisão variam conforme o critério utilizado, a característica avaliada, os tratamentos avaliados, o manejo e o conjunto de experimentos analisados. Essa diversidade de valores críticos (relacionado às espécies, às características, aos manejos) dificulta o controle ou o monitoramento da qualidade dos ensaios. Nestas publicações, há ênfase no estudo da distribuição da variável $\mathrm{CV}$, da definição do número de classes de precisão e do método de se obter os valores críticos destas classes.

A estatística diferença mínima significativa pelo teste de Tukey, em porcentagem da média (DMS), com propriedades semelhantes ao $\mathrm{CV}$, foi usada por Lúcio et al. (1999). Neste estudo, foram usadas cinco classes de precisão: muito alta ( $5 \%$ dos menores $\mathrm{CV}$ ); alta (entre 5 e $25 \%$ dos $\mathrm{CV}$ mais baixos); média (50\% centrais); baixa (entre 5 e $25 \%$ dos $\mathrm{CV}$ mais altos); e muito baixa (5\% dos maiores $\mathrm{CV}$ ), com a distribuição normal dos $\mathrm{CV}$.

Casos de experimentos com precisão média, alta ou muito alta não são preocupantes. O problema de se decidir pelo descarte ou não de um experimento está nos casos em que, apesar de não ter ocorrido nenhum fato relevante durante a sua condução que justifique tal procedimento, a precisão é muito baixa (CV muito alto) e quando, além disso, o experimento é de efeito não significativo para tratamento.

Parece haver consenso, entre os pesquisadores, sobre o uso do CV como medida da precisão experimental. Além disso, são comuns os descartes de experimentos com baixa precisão. No entanto, a indisponibilidade de tabelas de classificação, para cada característica observada, dificulta a classificação de experimentos quanto à precisão experimental. Nesse sentido, outras estatísticas como o coeficiente de herdabilidade (Cruz, 2001), coeficiente de determinação, valor do teste F para cultivar e índice de Fasoulas (Fasoulas, 1983) merecem ser investigadas sobre a possibilidade de serem utilizadas, no estabelecimento de valores críticos, para o controle ou o monitoramento da qualidade dos experimentos.

O objetivo deste trabalho foi avaliar estatísticas para a classificação da precisão experimental de ensaios de competição de cultivares de milho (Zea mays L.), em relação à produtividade de grãos.

\section{Material e Métodos}

Foram usados dados de produtividade de grãos de milho, corrigidos a 13\% de umidade, de 101 ensaios de competição de cultivares de milho (Zea mays L.), realizados no Estado do Rio Grande do Sul, nos anos agrícolas 2002/2003, 2003/2004 e 2004/2005, e obtidos em relatórios e atas das Reuniões Técnicas Anuais do Milho.

Os 101 ensaios foram classificados em 12 grupos de experimentos, conforme a categoria (estadual e

Tabela 1. Número de ensaios e número de cultivares de milho avaliadas nos anos agrícolas de 2002/2003, 2003/2004 e 2004/2005, em diferentes grupos de experimentos.

\begin{tabular}{|c|c|c|c|c|c|c|c|}
\hline \multicolumn{3}{|c|}{ Grupos de experimentos } & \multirow{2}{*}{$\begin{array}{c}\text { Número de ensaios } \\
\text { (locais) }\end{array}$} & \multicolumn{4}{|c|}{ Número de cultivares } \\
\hline Categoria $^{(1)}$ & Ciclo & Ano agrícola & & $\mathrm{HS}^{(2)}$ & $\mathrm{HT}^{(3)}$ & $\mathrm{HD}^{(4)}$ & Total \\
\hline Estadual & Precoce & $2002 / 2003$ & 10 & 13 & 11 & 12 & 36 \\
\hline Estadual & Superprecoce & $2002 / 2003$ & 9 & 6 & 3 & 2 & 11 \\
\hline Indicado & Precoce & $2002 / 2003$ & 6 & 12 & 8 & 7 & 27 \\
\hline Indicado & Superprecoce & $2002 / 2003$ & 5 & 7 & 6 & 5 & 18 \\
\hline Estadual & Precoce & $2003 / 2004$ & 13 & 17 & 12 & 11 & 40 \\
\hline Estadual & Superprecoce & $2003 / 2004$ & 12 & 6 & 3 & 0 & 9 \\
\hline Indicado & Precoce & $2003 / 2004$ & 7 & 11 & 9 & 6 & 26 \\
\hline Indicado & Superprecoce & $2003 / 2004$ & 7 & 7 & 6 & 3 & 16 \\
\hline Estadual & Precoce & $2004 / 2005$ & 11 & 13 & 12 & 7 & 32 \\
\hline Estadual & Superprecoce & $2004 / 2005$ & 11 & 10 & 5 & 2 & 17 \\
\hline Indicado & Precoce & $2004 / 2005$ & 5 & 16 & 6 & 8 & 30 \\
\hline Indicado & Superprecoce & $2004 / 2005$ & 5 & 5 & 4 & 3 & 12 \\
\hline Total & & & 101 & 123 & 85 & 66 & 274 \\
\hline
\end{tabular}

(1) $\mathrm{Na}$ categoria dos ensaios estaduais, as cultivares avaliadas foram as não indicadas aos produtores; as indicadas foram estudadas na categoria dos ensaios indicados. ${ }^{(2)}$ Híbrido Simples. ${ }^{(3)}$ Híbrido Triplo. ${ }^{(4)}$ Híbrido Duplo. 
indicado), o ciclo (precoce e superprecoce) e o ano agrícola (2002/2003, 2003/2004 e 2004/2005) (Tabela 1). Em todos os ensaios, as cultivares foram avaliadas segundo o delineamento de blocos ao acaso, com três repetições. As unidades experimentais foram constituídas de duas fileiras com $5 \mathrm{~m}$ de comprimento, espaçadas em $0,8 \mathrm{~m}$. As semeaduras foram realizadas, a fim de se obter populações finais de 55 mil e 65 mil plantas por hectare, respectivamente, em relação aos ensaios dos ciclos precoce e superprecoce.

Em relação à produtividade de grãos de milho, foram feitas análises de variância, para cada um dos 101 ensaios, e calculadas as estatísticas quadrado médio de bloco $\left(\mathrm{QM}_{\mathrm{B}}\right)$, quadrado médio de cultivar $\left(\mathrm{QM}_{\mathrm{C}}\right)$, quadrado médio do erro $\left(\mathrm{QM}_{\mathrm{E}}\right)$, valor do teste $\mathrm{F}$ para cultivar $(\mathrm{Fc})$, nível mínimo de significância do teste $\mathrm{F}$ para cultivar (nms), média geral do ensaio ( $\hat{\mathrm{m}})$, coeficiente de variação $(\mathrm{CV})$, variância genotípica $\left(\mathrm{V}_{\mathrm{GEN}}\right)$ e herdabilidade $\left(\mathrm{h}^{2}\right)$ (Cruz, 2001). Para a comparação de médias do efeito de cultivar, foi aplicado o teste de Tukey a 5\% de probabilidade.

A seguir, em cada ensaio, estimou-se a diferença mínima significativa entre as cultivares pelo teste de Tukey, a $5 \%$ de probabilidade $(\Delta)$, por meio da expressão: $\Delta=\mathrm{q}_{\alpha\left(\mathrm{n} ; \mathrm{GL}_{\mathrm{E}}\right)}\left(\mathrm{QM}_{\mathrm{E}} / \mathrm{J}\right)^{0,5}$, na qual $\mathrm{q}_{\alpha(\mathrm{n} ; \mathrm{GL}} \mathrm{E}_{\mathrm{E}}$ é o valor crítico para o uso do teste de Tukey; $n$ é o número de cultivares; $\mathrm{GL}_{\mathrm{E}}$ é o número de graus de liberdade do erro; $\mathrm{QM}_{\mathrm{E}}$ é a estimativa do erro experimental; J é o número de repetições. A diferença mínima significativa entre as cultivares, pelo teste de Tukey, expresso em porcentagem da média (DMS), foi obtida por $\mathrm{DMS}=100 \Delta / \hat{\mathrm{m}}$, em que $\hat{\mathrm{m}}$ é a estimativa da média geral do ensaio. Determinou-se, para cada ensaio, o valor da estatística $\mathrm{R}^{2}$, neste trabalho designada por coeficiente de determinação, calculada pela expressão $\mathrm{R}^{2}=\mathrm{QM}_{\mathrm{C}} /\left(\mathrm{QM}_{\mathrm{C}}+\mathrm{QM}_{\mathrm{E}}\right)$, e a amplitude total das médias $(\mathrm{H})$, estimada pela diferença entre as médias da cultivar com maior produtividade e a com menor produtividade ( $\mathrm{H}$ = média máxima - média mínima).

Definiu-se, a seguir, para cada ensaio, o índice de diferenciação de Fasoulas (IF) (Fasoulas, 1983), estimado pela expressão:

$\mathrm{IF}=200 \sum_{\mathrm{i}=1}^{\mathrm{n}} \mathrm{m}_{\mathrm{i}} /[\mathrm{n}(\mathrm{n}-1)]$

em que n é o número total de cultivares; $\mathrm{m}_{\mathrm{i}}$ é o número de médias que a i-ésima cultivar supera, estatisticamente, após a aplicação do teste de Tukey, fornecendo o porcentual de diferenças estatísticas entre as médias, que o método de comparação múltipla de médias (Tukey) consegue detectar.

Assim, foram obtidas 14 estatísticas $\left(\mathrm{QM}_{\mathrm{B}}, \mathrm{QM}_{\mathrm{C}}\right.$, $\mathrm{QM}_{\mathrm{E}}, \mathrm{Fc}, \mathrm{nms}, \hat{\mathrm{m}}, \mathrm{CV}, \mathrm{V}_{\mathrm{GEN}}, \mathrm{h}^{2}, \Delta, \mathrm{DMS}, \mathrm{R}^{2}, \mathrm{H}$ e IF) em cada ensaio, que foram utilizadas para avaliar a eficiência do CV como estatística, para a classificação da precisão experimental de ensaios de competição de cultivares de milho. Para isso, inicialmente, para cada estatística, foram calculados os valores mínimo, máximo, as medidas de tendência central (média e mediana), a medida de dispersão (desvio-padrão), a assimetria e a curtose, e foi realizado o teste de normalidade de Lilliefors (Campos, 1983). Após isso, fez-se análise de correlação linear de Pearson, entre as estatísticas, e sua significância foi verificada por meio do teste $t$ de Student. A seguir, realizou-se o diagnóstico de multicolinearidade (Cruz, 2001; Cruz \& Carneiro, 2003) e a análise de trilha ("path analysis") das variáveis principais, candidatas a medidas de precisão (CV, DMS, $\mathrm{h}^{2}, \mathrm{R}^{2}, \mathrm{Fc}$ e IF), em função das variáveis explicativas $\left(\mathrm{QM}_{\mathrm{B}}, \mathrm{QM}_{\mathrm{C}}, \mathrm{QM}_{\mathrm{E}}, \hat{\mathrm{m}}\right.$ e H).

Foram efetuadas as análises e obtidas as estimativas com o auxílio do programa GENES (Cruz, 2001).

\section{Resultados e Discussão}

O efeito de cultivar foi significativo $(\mathrm{p} \leq 0,05)$ em 93 ensaios $(92,08 \%)$, o que mostra que houve discriminação de cultivares quanto à produtividade de grãos de milho. Nos demais oito ensaios, não houve diferença significativa entre as cultivares. Do ponto de vista de discriminação de cultivares, por algum critério de controle de qualidade, somente estes últimos ensaios poderiam ser descartados. No entanto, este descarte deve ser visto com cautela, pois o fato de não serem detectadas diferenças significativas entre cultivares pode ser atribuído a sua inexistência ou ao erro experimental muito alto. Ao rejeitar a hipótese $\mathrm{H}_{0}$, afirma-se que há diferença significativa entre pelo menos um contraste de médias, com um erro tipo I de 5\%, de modo que há $5 \%$ de probabilidade de se tomar uma decisão errada. No entanto, a afirmação de não rejeitar $\mathrm{H}_{0}$, está associada ao erro tipo II, e neste caso, a probabilidade de se errar ao tomar esta decisão é desconhecida. Portanto, poderia ocorrer de se aceitar a não existência de diferenças entre as cultivares, mas ainda assim elas existirem.

O coeficiente de variação (CV), nos 101 ensaios de competição de cultivares de milho, em relação à 
produtividade de grãos, oscilou entre 5,04 e 21,80\%, e a diferença mínima significativa entre as cultivares, pelo teste de Tukey expresso em porcentagem da média a $5 \%$ de probabilidade (DMS), foi entre 15,49 e $70,61 \%$ (Tabela 2). Conforme classificação dos CV (Lúcio et al., 1999), esses ensaios enquadraram-se na faixa de alta à baixa precisão, e pela DMS de alta à muito baixa precisão. Ensaios com menor precisão (maiores valores de CV e DMS), a princípio, poderiam ser descartados.

Dos 101 ensaios, sete apresentaram CV $>20 \%$; destes, dois não apresentaram efeito significativo de cultivar, e cinco apresentaram efeito significativo, ou seja, $71 \%$ dos ensaios com $\mathrm{CV}>20 \%$ que, a princípio, poderiam ser descartados, apresentaram diferença entre cultivares, logo seus resultados não devem ser desconsiderados.

Três ensaios apresentaram os valores: $\mathrm{Fc}, 0,82,0,93$ e 0,99 ; CV, 21,14, 16,29 e 15,33\%; DMS, 65,04, 53,8 e $50,65 \%$. Nestes três experimentos, o valor do quadrado médio de cultivar $\left(\mathrm{QM}_{\mathrm{C}}\right)$ foi inferior ao quadrado médio do erro $\left(\mathrm{QM}_{\mathrm{E}}\right)$. A menor variância entre cultivares, em relação à variância entre as repetições dentro de cultivar, é inesperada e pode, provavelmente, ser explicada por problemas de amostragem (Barbin, 1998), por erro de especificação do modelo (alguma ocorrência estranha relevante não levada em conta, por exemplo) ou, ainda, por inexistência de diferenças de efeitos das cultivares no ambiente particular. Apesar disso, ao adotar o critério do $\mathrm{CV}$, dois ensaios estariam classificados em média precisão (Pimentel-Gomes, 1990; Lúcio et al., 1999). Os valores de Fc inferiores a um explicam a variância genotípica $\left(\mathrm{V}_{\mathrm{GEN}}\right)$ e a herdabilidade $\left(\mathrm{h}^{2}\right)$ negativa (Tabela 2).

De acordo com o teste de Lilliefors (Campos, 1983), das 14 estatísticas, apenas o CV, a DMS e a amplitude das médias $(\mathrm{H})$ ajustaram-se à distribuição normal $(\mathrm{p}>0,05)$ (Tabela 2). Valores de assimetria próximos de zero e de curtose próximos de três reforçam o comportamento normal da distribuição dessas estatísticas. Estes resultados confirmam que é adequado o estabelecimento de limites de classe de precisão experimental, das estatísticas CV e DMS, baseados na distribuição normal, como também foi constatado, por Lúcio et al. (1999), para os casos do CV e DMS.

Houve correlação linear significativa $(\mathrm{p} \leq 0,01)$ entre CV e DMS $(r=0,99)$ o que indica que a utilização de ambas, na classificação da precisão experimental, fornece informações de mesma natureza ou redundantes (Tabela 3). Lúcio et al. (1999) citam que a DMS é uma estatística adequada para a classificação dos experimentos, por possuir a mesma distribuição do $\mathrm{CV}$ e melhor noção quantitativa da estimativa da precisão. Também, a relação entre CV e DMS é, aproximadamente, DMS $=2,7 \mathrm{CV}$, e a correlação positiva é quase perfeita (Lúcio \& Storck, 1998).

Tabela 2. Mínimo, máximo, média, mediana, desvio-padrão, assimetria, curtose e teste de Lilliefors, das estatísticas de 101 ensaios de competição de cultivares de milho, em relação à produtividade de grãos.

\begin{tabular}{|c|c|c|c|c|c|c|c|c|}
\hline Estatística $^{(1)}$ & Mínimo & Máximo & Média & Mediana & Desvio-padrão & Assimetria $^{(2)}$ & Curtose $_{*}^{(3)}$ & Lilliefors \\
\hline $\mathrm{QM}_{\mathrm{B}}$ & 3.978 & 36.345 .587 & 4.135 .527 & 1.372 .230 & 7.078 .257 & $2,97^{*}$ & $12,38_{\mathrm{ns}}^{*}$ & $0,279713^{*}$ \\
\hline $\mathrm{QM}_{\mathrm{C}}$ & 782.615 & 9.256 .296 & 3.690 .983 & 3.075 .532 & 1.918 .262 & $0,98^{*}$ & $3,36_{*}^{\text {ns }}$ & $0,144609^{*}$ \\
\hline $\mathrm{QM}_{\mathrm{E}}$ & 198.944 & 3.105 .658 & 829.638 & 626.457 & 587.585 & $1,75^{*}$ & $6,34_{\mathrm{ns}}^{*}$ & $0,168906^{*}$ \\
\hline $\mathrm{Fc}$ & 0,82 & 18,88 & 5,90 & 4,89 & 3,86 & $1,26^{*}$ & $4,35_{*}^{\mathrm{ns}}$ & $0,149390^{*}$ \\
\hline $\mathrm{nms}$ & 0,00 & 0,66 & 0,03 & 0,00 & 0,11 & $4,40^{*}$ & $21,97_{*}^{*}$ & $0,411913^{*}$ \\
\hline Média & $2.328,21$ & $10.968,40$ & $6.963,20$ & $6.992,59$ & $2.258,98$ & $0,00^{\mathrm{ns}}$ & 1,82 & $0,096151^{*}$ \\
\hline $\mathrm{CV}$ & 5,04 & 21,80 & 13,25 & 13,06 & 4,24 & $0,08^{\text {ns }}$ & $2,10^{\mathrm{ns}}$ & $0,088622^{\mathrm{ns}}$ \\
\hline $\mathrm{V}_{\mathrm{GEN}}$ & -161.197 & 2.824 .852 & 953.782 & 797.007 & 629.085 & $1,00^{*}$ & $3,76_{*}^{\mathrm{ns}}$ & $0,138552^{*}$ \\
\hline$h^{2}$ & $-0,22$ & 0,95 & 0,73 & 0,80 & 0,22 & $-2,35^{*}$ & $9,32 *$ & $0,201908^{*}$ \\
\hline$\Delta$ & $1.372,10$ & $5.820,67$ & $2.705,25$ & $2.423,07$ & 921,44 & $1,07^{*}$ & $3,90_{*}^{\mathrm{ns}}$ & $0,141140_{\mathrm{ns}}^{*}$ \\
\hline DMS & 15,49 & 70,61 & 41,46 & 41,31 & 13,61 & $0,09^{\text {ns }}$ & $2,03 *$ & 0,076427 \\
\hline $\mathrm{R}^{2}$ & 0,45 & 0,95 & 0,81 & 0,83 & 0,11 & $-1,31^{*}$ & $4,81_{n \text { N }}^{*}$ & $0,125541^{*}$ \\
\hline Amplitude & $1.315,56$ & $6.610,44$ & $4.111,15$ & $3.957,28$ & $1.189,62$ & $0,20^{\mathrm{ns}}$ & $2,51_{\mathrm{ns}}^{\mathrm{ns}}$ & $0,080947^{\mathrm{ns}}$ \\
\hline IF & 0,00 & 43,64 & 11,31 & 8,27 & 10,46 & $0,98^{*}$ & $3,21^{\mathrm{ns}}$ & $0,160663^{*}$ \\
\hline
\end{tabular}

${ }^{(1)} \mathrm{QM}_{\mathrm{B}}$ : quadrado médio de bloco; $\mathrm{QM}_{\mathrm{C}}$ : quadrado médio de cultivar; $\mathrm{QM}_{\mathrm{E}}$ : quadrado médio do erro; Fc: valor do teste $\mathrm{F}$ para cultivar; nms: nível mínimo de significância do teste $\mathrm{F}$ para cultivar; Média: média geral do ensaio; $\mathrm{CV}$ : coeficiente de variação, em porcentagem; $\mathrm{V}_{\mathrm{GEN}}$ : variância genotípica; $\mathrm{h}^{2}$ : herdabilidade; $\Delta$ : diferença mínima significativa entre as cultivares, pelo teste de Tukey a $5 \%$ de probabilidade; DMS: diferença mínima significativa entre as cultivares, pelo teste de Tukey, expresso em porcentagem da média, a 5\% de probabilidade; $\mathrm{R}^{2}=\mathrm{QM}_{\mathrm{C}} /\left(\mathrm{QM}_{\mathrm{C}}+\mathrm{QM}_{\mathrm{E}}\right)$; Amplitude: amplitude das médias; IF: índice de diferenciação de Fasoulas (Fasoulas, 1983). ${ }^{(2)}$ A diferença significativa indicada é em relação a zero,

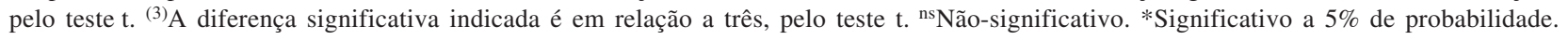


Houve associação linear negativa entre CV e a média $(\hat{\mathrm{m}})(\mathrm{r}=-0,61)$ e entre DMS e $\hat{\mathrm{m}}(\mathrm{r}=-0,60)$, o que indica que os ensaios com maior produtividade de grãos apresentaram menores CV e DMS (Tabela 3). Assim, o uso dessas estatísticas, como critério de classificação desses 101 ensaios, quanto à precisão experimental, é inadequado, pois elas estão sendo influenciadas pela média que, nos 101 ensaios estudados, oscilou entre $2.328,21 \mathrm{e} 10.968,40 \mathrm{~kg} \mathrm{ha}^{-1}$. A amplitude entre as médias $\left(8.640,19 \mathrm{~kg} \mathrm{ha}^{-1}\right)$ sugere que essa correlação seja esperada e explicada, parcialmente, pela propriedade de esperança matemática, pois ao somar-se uma constante à uma variável, a nova média passa a ser somada a essa constante, a variância não se altera, e o CV nessas condições é reduzido. Assim, numa rede de ensaios, ao se fornecerem melhores condições (adubação, irrigação, manejo) às plantas de um determinado experimento, soma-se, aproximadamente, uma constante à média de produtividade de grãos e, como consequência, esse ensaio tende a um CV menor, afirmação que está de acordo com Lopes \& Storck (1998).

Comprova-se que o CV e a DMS são estatísticas que servem para comparar a precisão experimental de experimentos com média semelhante. No entanto, após a separação desses ensaios em duas classes de produtividade de grãos $\left(<6.000 \mathrm{~kg} \mathrm{ha}^{-1} \mathrm{e} \geq 6.000 \mathrm{~kg} \mathrm{ha}^{-1}\right)$, apesar de em menor magnitude, continuou existindo correlação negativa entre $\mathrm{CV}$ e $\hat{\mathrm{m}}$, com $\mathrm{r}=-0,54$ $(p \leq 0,01)$ e $r=-0,47(p \leq 0,01)$, respectivamente, para essas duas classes de produtividade de grãos. Então, pode-se inferir que a média está influenciando o valor do CV e DMS, mesmo em classes de menor amplitude de variação entre médias de cultivares. Portanto, para se classificar a precisão experimental de ensaios pelas estatísticas CV e DMS, há necessidade de tabelas específicas, por classes de produtividade de grãos. A pouca disponibilidade de tabelas desse tipo dificulta essa classificação. Então, a investigação de estatísticas que independem da média do experimento é necessária, a fim de facilitar a classificação de experimentos em relação a qualquer variável.

As estatísticas herdabilidade $\left(\mathrm{h}^{2}\right)$, coeficiente de determinação $\left(\mathrm{R}^{2}\right)$, expresso por $\mathrm{R}^{2}=\mathrm{QM}_{\mathrm{C}}$ l $\left(\mathrm{QM}_{\mathrm{C}}+\mathrm{QM}_{\mathrm{E}}\right)$, valor calculado do teste $\mathrm{F}$ para cultivar $(\mathrm{Fc})$ e o índice de diferenciação de Fasoulas (IF) (Fasoulas, 1983), mesmo não tendo as propriedades da distribuição normal (Tabela 3), merecem ser investigadas como possíveis candidatas a estatísticas de classificação da precisão experimental e auxiliadoras na obtenção de algum critério de estabelecimento de valores críticos de controle da qualidade.

A ausência de correlação linear entre a média e as estatísticas $\mathrm{h}^{2}(\mathrm{r}=-0,12), \mathrm{R}^{2}(\mathrm{r}=-0,08), \mathrm{Fc}(\mathrm{r}=0,12)$ e IF $(r=0,17)$ as credencia como, provavelmente, adequadas a classificar a precisão experimental. A forte

Tabela 3. Coeficientes de correlação de Pearson, entre as estatísticas de 101 ensaios de competição de cultivares de milho, em relação à produtividade de grãos.

\begin{tabular}{|c|c|c|c|c|c|c|c|c|c|c|c|c|c|}
\hline Estatística $^{(1)}$ & $\mathrm{QM}_{\mathrm{C}}$ & $\mathrm{QM}_{\mathrm{E}}$ & $\mathrm{Fc}$ & $\mathrm{nms}$ & Média & $\mathrm{CV}$ & $\mathrm{V}_{\mathrm{GEN}}$ & $h^{2}$ & $\Delta$ & DMS & $\mathrm{R}^{2}$ & Amplitude & IF \\
\hline $\mathrm{QM}_{\mathrm{B}}$ & $-0,04^{\mathrm{ns}}$ & $0,23^{\mathrm{ns}}$ & $-0,24^{\mathrm{ns}}$ & $0,25^{\mathrm{ns}}$ & $0,23^{\mathrm{ns}}$ & $-0,04^{\text {ns }}$ & $-0,11^{\mathrm{ns}}$ & $-0,29^{* *}$ & $0,25^{\mathrm{ns}}$ & $-0,04^{\mathrm{ns}}$ & $-0,30^{* *}$ & $-0,05^{\mathrm{ns}}$ & $-0,23^{\mathrm{ns}}$ \\
\hline $\mathrm{QM}_{\mathrm{C}}$ & & $0,21^{\mathrm{ns}}$ & $0,52^{* *}$ & $-0,22^{\text {ns }}$ & $0,49^{* *}$ & $-0,26^{* *}$ & $0,95^{* *}$ & $0,37^{* *}$ & $0,24^{\mathrm{ns}}$ & $-0,25^{\mathrm{ns}}$ & $0,42^{* *}$ & $0,86^{* *}$ & $0,52^{* *}$ \\
\hline $\mathrm{QM}_{\mathrm{E}}$ & & & $-0,54^{* *}$ & $0,50^{* *}$ & $0,41^{* *}$ & $0,42^{* *}$ & $-0,10^{\mathrm{ns}}$ & $-0,71^{* *}$ & $0,98^{* *}$ & $0,42^{* *}$ & $-0,72^{* *}$ & $0,26^{* *}$ & $-0,51^{* *}$ \\
\hline $\mathrm{Fc}$ & & & & $-0,33^{* *}$ & $0,12^{\text {ns }}$ & $-0,61^{* *}$ & $0,69^{* *}$ & $0,69^{* *}$ & $-0,56^{* *}$ & $-0,60^{* *}$ & $0,80^{* *}$ & $0,42^{* *}$ & $0,95^{* *}$ \\
\hline $\mathrm{nms}$ & & & & & $0,16^{\mathrm{ns}}$ & $0,22^{\text {ns }}$ & $-0,38^{* *}$ & $-0,85^{* *}$ & $0,45^{* *}$ & $0,21^{\mathrm{ns}}$ & $-0,72^{* *}$ & $-0,24^{\mathrm{ns}}$ & $-0,29^{* *}$ \\
\hline Média & & & & & & $-0,61^{* *}$ & $0,37^{* *}$ & $-0,12^{\mathrm{ns}}$ & $0,41^{* *}$ & $-0,60^{* *}$ & $-0,08^{\mathrm{ns}}$ & $0,46^{* *}$ & $0,17^{\mathrm{ns}}$ \\
\hline $\mathrm{CV}$ & & & & & & & $-0,40^{* *}$ & $-0,46^{* *}$ & $0,42^{* *}$ & $0,99^{* *}$ & $-0,53^{* *}$ & $-0,22^{\text {ns }}$ & $-0,63^{* *}$ \\
\hline $\mathrm{V}_{\mathrm{GEN}}$ & & & & & & & & $0,60^{* *}$ & $-0,06^{\mathrm{ns}}$ & $-0,39^{* *}$ & $0,65^{* *}$ & $0,79^{* *}$ & $0,68^{* *}$ \\
\hline$h^{2}$ & & & & & & & & & $-0,68^{* *}$ & $-0,46^{* *}$ & $0,98^{* *}$ & $0,32^{* *}$ & $0,66^{* *}$ \\
\hline$\Delta$ & & & & & & & & & & $0,45^{* *}$ & $-0,71^{* *}$ & $0,33^{* *}$ & $-0,55^{* *}$ \\
\hline DMS & & & & & & & & & & & $-0,53^{* *}$ & $-0,17^{\text {ns }}$ & $-0,63^{* *}$ \\
\hline & & & & & & & & & & & & $0,35^{* *}$ & $0,78^{* *}$ \\
\hline Amplitude & & & & & & & & & & & & & $0,35^{* *}$ \\
\hline
\end{tabular}

${ }^{(1)} \mathrm{QM}_{\mathrm{B}}$ : quadrado médio de bloco; $\mathrm{QM}_{\mathrm{C}}$ : quadrado médio de cultivar; $\mathrm{QM}_{\mathrm{E}}$ : quadrado médio do erro; Fc: valor do teste $\mathrm{F}$ para cultivar; nms: nível mínimo de significância do teste $\mathrm{F}$ para cultivar; Média: média geral do ensaio; $\mathrm{CV}$ : coeficiente de variação, em porcentagem; $\mathrm{V}_{\mathrm{GEN}}$ : variância genotípica; $\mathrm{h}^{2}$ : herdabilidade; $\Delta$ : diferença mínima significativa entre as cultivares, pelo teste de Tukey, a 5\% de probabilidade; DMS: diferença mínima significativa entre as cultivares, pelo teste de Tukey expresso em porcentagem da média, a 5\% de probabilidade; $\mathrm{R}^{2}=\mathrm{QM}_{\mathrm{C}}\left(\mathrm{QM}_{\mathrm{C}}+\mathrm{QM}_{\mathrm{E}}\right)$;

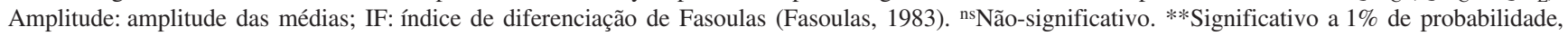
pelo teste t, com 99 graus de liberdade. 
associação linear positiva, entre $h^{2}$ e $R^{2}(r=0,98)$ e entre Fc e IF $(r=0,95)$, revela semelhança entre as estatísticas, e no caso de serem adequadas não há necessidade de se optar por ambas, podendo, como sugestão, ser usado o $\mathrm{R}^{2}$ e Fc pela facilidade de cálculo (Tabela 3 ).

As estatísticas nível mínimo de significância do teste F para cultivar (nms) (alta correlação com $\mathrm{h}^{2} \mathrm{e} \mathrm{R}^{2}$ ), variância genotípica $\left(\mathrm{V}_{\mathrm{GEN}}\right)$ (alta correlação com $\mathrm{QM}_{\mathrm{C}}$, $\mathrm{Fc}, \mathrm{h}^{2}, \mathrm{R}^{2}, \mathrm{H}$ e IF) e diferença mínima significativa entre as cultivares, pelo teste de Tukey, a 5\% de probabilidade $(\Delta)$ (alta correlação com $\mathrm{QM}_{\mathrm{E}}, \mathrm{Fc}, \mathrm{h}^{2}, \mathrm{R}^{2}$ e IF), foram eliminadas da análise de trilha, por causarem colinearidade na matriz das correlações (Cruz, 2001; Cruz \& Carneiro, 2003).

Com a eliminação das variáveis nms, $\mathrm{V}_{\mathrm{GEN}} \mathrm{e} \Delta$, o número de condição oscilou entre 21 [ $F$ c função $\left(\mathrm{QM}_{\mathrm{B}}\right.$, $\mathrm{QM}_{\mathrm{C}}, \mathrm{QM}_{\mathrm{E}}, \hat{\mathrm{m}}$ e $\left.\mathrm{H}\right)$ ] e 71 [CV função $\left(\mathrm{QM}_{\mathrm{B}}, \mathrm{QM}_{\mathrm{C}}\right.$, $\mathrm{QM}_{\mathrm{E}}$, $\hat{\mathrm{m}}$ e $\left.\mathrm{H}\right)$ ], o que classifica a matriz de correlações como de colinearidade baixa e permite a realização adequada da análise de trilha (Cruz, 2001; Cruz \& Carneiro, 2003).

Assim, foram realizadas análises de trilha das variáveis principais (CV, DMS, $h^{2}, \mathrm{R}^{2}, \mathrm{Fc}$ e IF), em função das variáveis explicativas $\mathrm{QM}_{\mathrm{B}}, \mathrm{QM}_{\mathrm{C}}, \mathrm{QM}_{\mathrm{E}}, \hat{\mathrm{m}}$ e H (Tabela 4).

As estatísticas CV, DMS, $\mathrm{h}^{2}, \mathrm{R}^{2}$, Fc e IF apresentaram correlação linear com o $\mathrm{QM}_{\mathrm{E}}$ e efeito direto de mesmo sinal e magnitude semelhante, o que evidencia que todas têm associação com a variância residual (Tabela 4). Assim, pode-se inferir que, a princípio, todas são adequadas para a classificação da precisão experimental. No entanto, as estatísticas CV e DMS apresentaram correlação linear com a média e efeito direto de mesmo sinal e de magnitude semelhante, o que mostra que experimentos com maiores produtividades apresentam menor CV $(-0,9481)$ e DMS $(-0,9441)$. Esse fato, aliado à ausência de associação efeito direto do $\mathrm{QM}_{\mathrm{C}} \mathrm{com}$ as estatísticas CV $(0,1367)$ e DMS $(-0,0030)$, torna-as pouco adequadas para classificar esses ensaios (Tabela 4). Assim, o descarte de ensaios com maiores escores de CV e DMS eliminaria aqueles com menor média de produtividade de grãos, com maior variância residual, independentemente das diferenças entre cultivares $\left(\mathrm{QM}_{\mathrm{C}}\right)$.

As estatísticas $\mathrm{h}^{2}$ e $\mathrm{R}^{2}$ estão associadas ao $\mathrm{QM}_{\mathrm{C}}$, ao $\mathrm{QM}_{\mathrm{E}}$ e à $\mathrm{H}$ e não estão associadas ao $\mathrm{QM}_{\mathrm{B}} \mathrm{e}$ à média. As estatísticas Fc e IF estão associadas ao $\mathrm{QM}_{\mathrm{C}}$ e ao $\mathrm{QM}_{\mathrm{E}}$ e não estão associadas ao $\mathrm{QM}_{\mathrm{B}}$, à média e à $\mathrm{H}$. Esses resultados evidenciam vantagens das estatísticas $h^{2}, R^{2}$, Fc e IF, em relação ao CV e à DMS, ou seja, ensaios classificados como mais precisos por $\mathrm{h}^{2}, \mathrm{R}^{2}$, Fc e IF não dependeriam da média e estariam relacionados a menores variâncias residuais $\left(\mathrm{QM}_{\mathrm{E}}\right)$ e maiores variâncias entre tratamentos $\left(\mathrm{QM}_{\mathrm{C}}\right)$, o que é desejável do ponto de vista de precisão experimental. Tabelas de classificação da precisão experimental, a partir dessas estatísticas, servem para classificar precisão experimental de experimentos em geral. Assim, experimentos mais precisos (maiores escores de $h^{2}, R^{2}, F c$ e IF) estariam associados a menores variâncias residuais $\left(\mathrm{QM}_{\mathrm{E}}\right)$ e maiores variâncias genéticas $\left(\mathrm{QM}_{\mathrm{C}}\right)$, independentemente da média.

Assim, por estas estatísticas, os experimentos com alta variabilidade residual e baixa variabilidade genética entre cultivares tendem a ser eliminados, o que é adequado. Isso é particularmente importante, pois a tendência é classificar como experimentos mais precisos aqueles que têm maior variabilidade genética e menor variabilidade residual, portanto, aqueles que discriminam mais as cultivares, independentemente da média geral do experimento.

Entre as quatro últimas estatísticas $\left(\mathrm{h}^{2}, \mathrm{R}^{2}, \mathrm{Fc}\right.$ e IF), apesar da semelhança dos resultados na análise de trilha, $\mathrm{o}$ maior efeito direto do $\mathrm{QM}_{\mathrm{E}}$, sobre as estatísticas $\mathrm{h}^{2}(-0,8088)$ e $\mathrm{R}^{2}(-0,8374)$, caracteriza uma vantagem em relação às estatísticas Fc (-0,7023) e IF (-0,6622). No entanto, a menor magnitude do efeito direto do $\mathrm{QM}_{\mathrm{C}}$, sobre as estatísticas $h^{2}(0,3378)$ e $R^{2}(0,3983)$, caracteriza uma desvantagem em relação às estatísticas $\mathrm{Fc}(0,4898)$ e IF $(0,6890)$ (Tabela 4). Ainda, mesmo com menor magnitude, o efeito direto da $\mathrm{H}$ sobre as estatísticas $h^{2}(0,2597)$ e $R^{2}(0,2377)$ caracteriza outra vantagem destas, em relação às estatísticas $\mathrm{Fc}(0,1219)$ e IF $(-0,1586)$ que não demonstraram associação com a amplitude.

Do ponto de vista de classificação de precisão experimental, as estatísticas CV e DMS são adequadas para experimentos sob condições específicas, e as tabelas de classificação de precisão experimental devem ser feitas para cada variável e em intervalos menores. As estatísticas $\mathrm{h}^{2}, \mathrm{R}^{2}, \mathrm{Fc}$ e IF são adequadas para classificar precisão experimental em condições gerais, o que as torna vantajosas sobre as primeiras e, por serem estatísticas independentes da média, permitem o estabelecimento de tabelas de classificação para experimentos. Deve-se, no entanto, estudar algum critério para a definição de valores críticos, a fim de classificar a precisão dos experimentos, considerando-se que as estatísticas $\mathrm{h}^{2}, \mathrm{R}^{2}, \mathrm{Fc}$ e IF não seguem a distribuição normal. 
Tabela 4. Estimativa dos coeficientes de correlação de Pearson e respectivas estimativas dos efeitos diretos e indiretos das estatísticas $\mathrm{QM}_{\mathrm{B}}, \mathrm{QM}_{\mathrm{C}}, \mathrm{QM}_{\mathrm{E}}$, média e amplitude sobre as estatísticas $\mathrm{CV}, \mathrm{DMS}, \mathrm{h}^{2}, \mathrm{R}^{2}$, Fc e IF de 101 ensaios de competição de cultivares de milho, em relação à produtividade de grãos.

\begin{tabular}{|c|c|c|c|c|c|}
\hline Efeito & $\mathrm{QM}_{\mathrm{B}}$ & $\mathrm{QM}_{\mathrm{C}}$ & $\mathrm{QM}_{\mathrm{E}}$ & Média & Amplitude \\
\hline & & & Sobre CV & & \\
\hline Direto sobre CV & $-0,0109$ & 0,1367 & 0,8056 & $-0,9481$ & $-0,1153$ \\
\hline Indireto via $\mathrm{QM}_{\mathrm{B}}$ & & 0,0005 & $-0,0025$ & $-0,0025$ & 0,0006 \\
\hline Indireto via $\mathrm{QM}_{\mathrm{C}}$ & $-0,0059$ & & 0,0281 & 0,0671 & 0,1176 \\
\hline Indireto via $\mathrm{QM}_{\mathrm{E}}$ & 0,1827 & 0,1655 & & 0,3267 & 0,2112 \\
\hline Indireto via Média & $-0,2164$ & $-0,4658$ & $-0,3845$ & & $-0,4315$ \\
\hline Indireto via Amplitude & 0,0059 & $-0,0992$ & $-0,0302$ & $-0,0525$ & \\
\hline Total (Corr. Pearson) & $-0,0446^{\mathrm{ns}}$ & $-0,2624^{* *}$ & $0,4165^{* *}$ & $-0,6092^{* *}$ & $-0,2175^{\mathrm{ns}}$ \\
\hline \multirow[t]{2}{*}{ Coeficiente de determinação } & & & 0,9028 & & \\
\hline & \multicolumn{4}{|c|}{ Sobre DMS } & \\
\hline Direto sobre DMS & 0,0036 & $-0,0030$ & 0,7874 & $-0,9441$ & 0,0605 \\
\hline Indireto via $\mathrm{QM}_{\mathrm{B}}$ & & $-0,0002$ & 0,0008 & 0,0008 & $-0,0002$ \\
\hline Indireto via $\mathrm{QM}_{\mathrm{C}}$ & 0,0001 & & $-0,0006$ & $-0,0015$ & $-0,0026$ \\
\hline Indireto via $\mathrm{QM}_{\mathrm{E}}$ & 0,1786 & 0,1618 & & 0,3193 & 0,2064 \\
\hline Indireto via Média & $-0,2155$ & $-0,4638$ & $-0,3829$ & & $-0,4297$ \\
\hline Indireto via Amplitude & $-0,0031$ & 0,0521 & 0,0159 & 0,0276 & \\
\hline Total (Corr. Pearson) & $-0,0363^{\mathrm{ns}}$ & $-0,2531^{\mathrm{ns}}$ & $0,4206^{* *}$ & $-0,5979^{* *}$ & $-0,1656^{\mathrm{ns}}$ \\
\hline \multirow[t]{2}{*}{ Coeficiente de determinação } & & & 0,8863 & & \\
\hline & \multicolumn{4}{|c|}{ Sobre $h^{2}$} & \\
\hline Direto sobre $\mathrm{h}^{2}$ & $-0,0675$ & 0,3378 & $-0,8088$ & $-0,0577$ & 0,2597 \\
\hline Indireto via $\mathrm{QM}_{\mathrm{B}}$ & & 0,0029 & $-0,0153$ & $-0,0154$ & 0,0034 \\
\hline Indireto via $\mathrm{QM}_{\mathrm{C}}$ & $-0,0146$ & & 0,0694 & 0,1660 & 0,2907 \\
\hline Indireto via $\mathrm{QM}_{\mathrm{E}}$ & $-0,1835$ & $-0,1662$ & & $-0,3280$ & $-0,2120$ \\
\hline Indireto via Média & $-0,0132$ & $-0,0283$ & $-0,0234$ & & $-0,0263$ \\
\hline Indireto via Amplitude & $-0,0133$ & 0,2234 & 0,0681 & 0,1182 & \\
\hline Total (Corr. Pearson) & $-0,2920^{* *}$ & $0,3696^{* *}$ & $-0,7100^{* *}$ & $-0,1169^{\mathrm{ns}}$ & $0,3155^{* *}$ \\
\hline \multirow[t]{2}{*}{ Coeficiente de determinação } & & & 0,8075 & & \\
\hline & \multicolumn{4}{|c|}{ Sobre $\mathbf{R}^{2}$} & \\
\hline Direto sobre $\mathrm{R}^{2}$ & $-0,0734$ & 0,3983 & $-0,8374$ & $-0,0257$ & 0,2377 \\
\hline Indireto via $\mathrm{QM}_{\mathrm{B}}$ & & 0,0032 & $-0,0167$ & $-0,0168$ & 0,0038 \\
\hline Indireto via $\mathrm{QM}_{\mathrm{C}}$ & $-0,0172$ & & 0,0819 & 0,1957 & 0,3428 \\
\hline Indireto via $\mathrm{QM}_{\mathrm{E}}$ & $-0,1899$ & $-0,1721$ & & $-0,3396$ & $-0,2195$ \\
\hline Indireto via Média & $-0,0059$ & $-0,0126$ & $-0,0104$ & & $-0,0117$ \\
\hline Indireto via Amplitude & $-0,0122$ & $0,2045 *$ & 0,0623 & 0,1082 & \\
\hline Total (Corr. Pearson) & $-0,2986^{* *}$ & $0,4213^{* *}$ & $-0,7203^{* *}$ & $-0,0782^{\mathrm{ns}}$ & $0,3530^{* *}$ \\
\hline \multirow[t]{2}{*}{ Coeficiente de determinação } & & & 0,8788 & & \\
\hline & \multicolumn{4}{|c|}{ Sobre Fc } & \\
\hline Direto sobre Fc & $-0,0801$ & 0,4898 & $-0,7023$ & 0,1299 & 0,1219 \\
\hline Indireto via $\mathrm{QM}_{\mathrm{B}}$ & & 0,0035 & $-0,0182$ & $-0,0183$ & 0,0041 \\
\hline Indireto via $\mathrm{QM}_{\mathrm{C}}$ & $-0,0212$ & & 0,1006 & 0,2406 & 0,4214 \\
\hline Indireto via $\mathrm{QM}_{\mathrm{E}}$ & $-0,1593$ & $-0,1443$ & & $-0,2848$ & $-0,1841$ \\
\hline Indireto via Média & 0,0297 & 0,0638 & 0,0527 & & 0,0591 \\
\hline Indireto via Amplitude & $-0,0062$ & 0,1049 & 0,0320 & 0,0555 & \\
\hline Total (Corr. Pearson) & $-0,2371^{\mathrm{ns}}$ & $0,5177^{* *}$ & $-0,5352^{* *}$ & $0,1229^{\mathrm{ns}}$ & $0,4225^{* *}$ \\
\hline \multirow[t]{2}{*}{ Coeficiente de determinação } & & & 0,7159 & & \\
\hline & \multicolumn{4}{|c|}{ Sobre IF } & \\
\hline Direto sobre IF & $-0,0995$ & 0,6890 & $-0,6622$ & 0,1944 & $-0,1586$ \\
\hline Indireto via $\mathrm{QM}_{\mathrm{B}}$ & & 0,0043 & $-0,0226$ & $-0,0227$ & 0,0051 \\
\hline Indireto via $\mathrm{QM}_{\mathrm{C}}$ & $-0,0298$ & & 0,1416 & 0,3385 & 0,5929 \\
\hline Indireto via $\mathrm{QM}_{\mathrm{E}}$ & $-0,1502$ & $-0,1361$ & & $-0,2685$ & $-0,1736$ \\
\hline Indireto via Média & 0,0444 & 0,0955 & 0,0788 & & 0,0885 \\
\hline Indireto via Amplitude & 0,0081 & $-0,1365$ & $-0,0416$ & $-0,0722$ & \\
\hline Total (Corr. Pearson) & $-0,2270^{\mathrm{ns}}$ & $0,5162^{* *}$ & $-0,5059^{* *}$ & $0,1694^{\mathrm{ns}}$ & $0,3542^{* *}$ \\
\hline Coeficiente de determinação & & & 0,6900 & & \\
\hline
\end{tabular}




\section{Conclusões}

1. O coeficiente de variação e a diferença mínima significativa entre as cultivares, pelo teste de Tukey expresso em porcentagem da média, estão associados à média e à variância residual, e são estatísticas adequadas para a classificação de experimentos com média semelhante.

2. Os maiores valores das estatísticas herdabilidade, coeficiente de determinação, valor do teste $F$ para cultivar e índice de diferenciação de Fasoulas estão associados a maiores variabilidades genéticas e menores variâncias residuais e independem da média do ensaio; estas estatísticas são adequadas para a classificação da precisão de experimentos.

\section{Agradecimentos}

Ao CNPq, pelo auxílio financeiro; ao Eng. Agrôn. José Paulo Guadagnin, da Fundação Estadual de Pesquisa Agropecuária, pela coordenação dos ensaios de avaliação de cultivares de milho no Estado do Rio Grande do Sul; aos pesquisadores de outras instituições, pelo auxílio.

\section{Referências}

AMARAL, A.M.; MUNIZ, J.A.; SOUZA, M. Avaliação do coeficiente de variação como medida da precisão na experimentação com citros. Pesquisa Agropecuária Brasileira, v.32, p.1221-1225, 1997.

BARBIN, D. Componentes de variância: teoria e aplicações. 2.ed. Piracicaba: Fealq, 1998. 120p.

CAMPOS, H. Estatística experimental não-paramétrica. 4.ed. Piracicaba: Esalq, Departamento de Matemática e Estatística, 1983. 349 p.

CARVALHO, C.G.P.; ARIAS, C.A.A.; TOLEDO, J.F.F.; ALMEIDA, L.A.; KIIHL, R.A.S.; OLIVEIRA, M.F.; HIROMOTO, D.M.; TAKEDA, C. Proposta de classificação dos coeficientes de variação em relação à produtividade e altura da planta de soja. Pesquisa Agropecuária Brasileira, v.38, p.187-193, 2003.

CLEMENTE, A.L.; MUNIZ, J.A. Avaliação do coeficiente de variação em experimentos com gramíneas forrageiras. Ciência e Agrotecnologia, v.26, p.197-203, 2002.

COSTA, N.H.A.D.; SERAPHIN, J.C.; ZIMMERMANN, F.J.P. Novo método de classificação de coeficientes de variação para a cultura do arroz de terras altas. Pesquisa Agropecuária Brasileira, v.37, p.243-249, 2002.
CRUZ, C.D. Programa GENES: versão Windows: aplicativo computacional em genética e estatística. Viçosa: UFV, 2001. 648p.

CRUZ, C.D.; CARNEIRO, P.C.S. Modelos biométricos aplicados ao melhoramento genético. Viçosa: UFV, 2003. v.2, 585p.

ESTEFANEL, V.; PIGNATARO, I.A.B.; STORCK, L. Avaliação do coeficiente de variação de experimentos com algumas culturas agrícolas. In: SIMPÓSIO DE ESTATÍSTICA APLICADA À EXPERIMENTAÇÃO AGRONÔMICA, 2., 1987, Londrina. Anais. Londrina: Universidade Estadual de Londrina/Região Brasileira da Sociedade Internacional de Biometria, 1987. p.115-131.

FASOULAS, A.C. Rating cultivars and trials in applied plant breeding. Euphytica, v.32, p.939-943, 1983.

GARCIA, C.H. Tabelas para classificação do coeficiente de variação. Piracicaba: Ipef, 1989. 12p. (Circular técnica, 171).

JUDICE, M.G.; MUNIZ, J.A.; AQUINO, L.H.; BEARZOTI, E. Avaliação da precisão experimental em ensaios com bovinos de corte. Ciência e Agrotecnologia, v.26, p.1035-1040, 2002.

JUDICE, M.G.; MUNIZ, J.A.; CARVALHEIRO, R. Avaliação do coeficiente de variação na experimentação com suínos. Ciência e Agrotecnologia, v.23, p.170-173, 1999.

LIMA, L.L.; NUNES, G.H.S.; BEZERRA NETO, F. Coeficientes de variação de algumas características do meloeiro: uma proposta de classificação. Horticultura Brasileira, v.22, p.14-17, 2004.

LOPES, S.J.; STORCK, L. Heterogeneidade do solo sob diferentes adubações na cultura do milho. Ciência Rural, v.28, p.361-366, 1998.

LÚCIO, A.D.; STORCK, L. O manejo das culturas interfere no erro experimental. Pesquisa Agropecuária Gaúcha, v.5, p.311-316, 1999.

LÚCIO, A.D.; STORCK, L. Relação entre diferença mínima significativa e coeficiente de variação nos ensaios de competição de cultivares. Ciência Rural, v.28, p.225-228, 1998.

LÚCIO, A.D.; STORCK, L.; BANZATTO, D.A. Classificação dos experimentos de competição de cultivares quanto a sua precisão. Pesquisa Agropecuária Gaúcha, v.5, p.99-103, 1999.

LÚCIO, A.D.; STORCK, L.; MARCHEZAN, E. Verifique a qualidade de seu experimento. Lavoura Arrozeira, v.50, p.23-25, 1997.

PIMENTEL-GOMES, F. Curso de estatística experimental. 13.ed. Piracicaba: Nobel, 1990. 468p.

PIMENTEL-GOMES, F. O índice de variação: um substituto vantajoso do coeficiente de variação. Piracicaba: Ipef, 1991. 4p. (Circular técnica, 178).

SCAPIM, C.A.; CARVALHO, C.G.P. de; CRUZ, C.D. Uma proposta de classificação dos coeficientes de variação para a cultura do milho. Pesquisa Agropecuária Brasileira, v.30, p.683-686, 1995.

STORCK, L.; LÚCIO, A.D.; SANTOS, P.M.; CARVALHO, M.P.; CARDINAL, A.B.B. Precisão experimental em erva-mate (Ilex paraguariensis St.Hil.). Ciência Florestal, v.12, p.159-161, 2002.

Recebido em 28 de março de 2006 e aprovado em 11 de outubro de 2006 